Noman 2016, 34(2), 87-93

Revista de Psicologia, Ciències de l'Educació i de l'Esport

ISSN: 1138-3194

Copyright (c) 2016

www.revistaaloma.net

\title{
La evaluación de la transferencia de un programa de entrenamiento de la memoria
}

\author{
Cristina Vidal-Martí1 \& Carles Pérez-Testor ${ }^{2}$ \\ ${ }^{1}$ Fundació Pere Tarrés \\ ${ }^{2}$ Universitat Ramon Llull \\ Recibido: 4-5-2016 \\ Aceptado:15-9-2016
}

La evaluación de la transferencia de un programa de entrenamiento de la memoria

Resumen. Los programas de entrenamiento de la memoria son programas psicoeducativos dirigidos a personas adultas y mayores sin deterioro cognitivo. Su fin es entrenar la memoria y optimizar su rendimiento. El objetivo de este estudio es medir la transferencia de conocimientos del programa de memoria Si entrenamos, recordamos. Este programa se estructura en tres proyectos. Dicha investigación se centra propiamente en evaluar la transferencia del segundo proyecto con relación al primero.

Se escogió el modelo de evaluación de Kirkpatrick para evaluar la transferencia. En esta investigación de carácter descriptivo y exploratorio se administraron cuatro instrumentos ad hoc con la finalidad de medir los cuatro niveles establecidos en el modelo Kirkpatrick. Su administración tuvo lugar en dos etapas distintas al proceso. Participaron 29 personas mayores. Los resultados establecen que el 89.7\% de los dinamizadores supo explicar los contenidos, el 93\% realizó actividades facilitadoras del aprendizaje y un 69\% dio respuesta a las dudas planteadas por los participantes. Estos resultados fueron confirmados por los educadores.

Palabras clave: memoria; programas de transferencia; transferencia de la formación; gestión del conocimiento

\section{Assessment of information transfer of a memory training programme}

Summary. Memory training programmes are psychoeducational programmes aimed at adults and older people without any cognitive deterioration. Their goal is to entertain the memory and optimise its performance. The objective of this study was to measure the transfer of knowledge resulting from the memory programme «If we train, we remember». The programme consists of three projects. This study is specifically focussed on assessing the information transfer achieved in the second phase of the project and comparing it to what was attained in the first. Kirkpatrick's assessment model was chosen to assess the degree of transfer. In this descriptive, exploratory study, four ad hoc instruments were used to measure the four levels established by the Kirkpatrick model. They were administered at two different points in the process to the participants, 29 older people. The results showed that 89.7\% of the «dynamic leaders» were able to explain the contents, 93\% undertook activities that facilitated learning and $69 \%$ responded to the questions posed by the participants. These results were confirmed by the educators.

Keywords: Memory; transfer programmes; educational transfer; knowledge management

\author{
Correspondencia: \\ Cristina Vidal-Martí \\ Fundación Pere Tarrés \\ C/ Carolines, 10 \\ 08012 Barcelona \\ Teléfono: 934101602 \\ cristinavidal.vm@gmail.com
}




\section{Introducción}

\section{Los programas de entrenamiento de la memoria}

Los programas de entrenamiento de la memoria son acciones psicoeducativas dirigidas a personas adultas y mayores sin deterioro cognitivo (Pérez et al., 2015). $\mathrm{Su}$ principal objetivo es entrenar la memoria combinando el aprendizaje de diferentes estrategias de memoria y funciones cognitivas (Israel, 1992; Montejo, 2001; Delgado Losada, 2015).

Montejo, Montenegro, Reinoso, Andrés y Claver (2001) definen el entrenamiento de la memoria como «(...) adiestrar de una manera sistemática en el conocimiento, la utilización y el control de los procesos, estrategias, técnicas y vivencias implicadas en el funcionamiento de la memoria y en la mejora de su rendimiento (...)» (p. 332).

Los primeros programas de entrenamiento de la memoria aparecieron alrededor de los años setenta. Se dirigían mayoritariamente a personas con trastornos orgánicos cerebrales con el fin de estimular su capacidad cognitiva y promover la rehabilitación (Hernández Viadel \& Latorre Postigo, 2008). Sin embargo, a raíz de su consolidación, dichos programas se orientaron a personas mayores con problemas neurológicos y de manera más residual a otros colectivos. A finales de los años ochenta, se focalizaron en personas mayores, centrándose en procesos de envejecimiento saludable y excluyendo a población con patología, como por ejemplo los trastornos neurodegenerativos (Montejo, 2003).

El primer programa de memoria fue creado por Israel (1992). Planteó un modelo de intervención basado en la combinación de la acción pedagógica con la psicoterapéutica, con el fin de atender a las personas mayores de cincuenta años con quejas subjetivas de memoria. Este modelo estableció cuatro objetivos. El primero fue concienciar a los participantes de los mecanismos implicados en el funcionamiento de la memoria. El segundo, reducir la ansiedad y el estrés que experimenta la persona cuando aparecen los primeros trastornos de memoria. El tercero, favorecer la creación de nuevas actitudes gracias a una atención sostenida, una mayor receptividad y predisposición. Y el cuarto era aprender a utilizar estrategias que permitan compensar los efectos de los trastornos de la memoria.

La metodología del programa de entrenamiento propuesto por Israel se basa en la intervención grupal y los contenidos parten de los mecanismos implicados en el funcionamiento de la memoria.

Este método facilitó la introducción y la consolidación de los programas de entrenamiento de la memoria gracias a su estructuración por ser un programa organizado, coherente con las necesidades de las personas mayores, con el funcionamiento de la memoria y sus mecanismos implicados. Será a raíz de dicho programa que aparecen nuevas propuestas (VidalMartí, 2012).

En los últimos años, el número de programas de entrenamiento de la memoria ha aumentado (Baena Díez et al., 2009). El carácter preventivo y el aumento de la demanda por parte de las personas mayores contribuyó a que organismos de salud, de servicios sociales y organizaciones relacionadas con dichos entes apostaran por este tipo de programas y los organizaran de manera continua y con periodicidad regular.

En la actualidad, hay una gran variedad de programas que tienen como objetivo principal el entrenamiento de la memoria (Vidal-Martí, 2015). Sin embargo, estos programas difieren sustancialmente unos de otros en cuestiones básicas como son los objetivos, los contenidos, la metodología, la duración, la selección de los participantes, la función del educador y los niveles, entre otros (Novoa, Juárez \& Nebot, 2008).

Conscientes de la disparidad de los programas existentes nace el programa Si entrenamos, recordamos (Vidal-Martí, 2012). Se trata de un programa de entrenamiento de la memoria que responde a dos necesidades: la sistematización y la adaptación al público objetivo.

Durante años, los profesionales que imparten programas de entrenamiento de la memoria utilizan diferentes fuentes por la poca literatura con relación a los fundamentos teóricos y la escasez de actividades y recursos para el colectivo de personas mayores. A nuestro entender, era conveniente diseñar una programa de memoria que dotase a los profesionales de recursos desde un marco de conceptualización de la intervención; es decir, que la intervención se justificara a partir de fundamentos teóricos. Este proceso conllevó a una estructuración y sistematización de la actuación. En un primer momento, se inició un proceso de detección y análisis de las necesidades, continuó con una identificación de las mismas y prosiguió con su priorización y la planificación del proyecto. Este proceso se llevó a cabo hasta su implementación y evaluación (VidalMartí et al., 2013).

Una segunda necesidad era responder a las demandas del público objetivo: las personas mayores. Estas comentan que las actividades que plantean algunos programas de entrenamiento no se adecuan a sus necesidades del día a día, ya sea por ser excesivamente escolares, abstractas o porque no dan respuesta a las necesidades evolutivas.

\section{El programa de entrenamiento de la memoria}

El programa Si entrenamos, recordamos consta de tres proyectos interrelacionados entre sí y que tienen un orden establecido. El primero, denominado Entrenamiento de la memoria, es un proyecto psicoeducativo, estructurado, de duración determinada y dirigido por un profesional. Sus objetivos son cuatro: el primero, descubrir el placer de entrenar la memoria; el segundo, saber afrontar la pérdida de la memoria propia del envejecimiento saludable; el tercero promueve la transferencia de conocimientos, habilidades y técnicas en la vida cotidiana; y el cuarto enseña a utilizar estrategias y procesos cognitivos para optimizar la memoria. La metodología combina las actividades grupales e individuales y se trabajan contenidos teóricos que permiten reforzar la metamemoria. 
El segundo, Practica tu memoria, se diferencia del anterior por ser dinamizado por personas mayores voluntarias. Refuerza los objetivos y contenidos trabajados en el primer proyecto. Su metodología está orientada a trabajar con todo el grupo, el estilo de dinamización es menos dirigido y tiene una estructuración más flexible respecto al primero. Los dinamizadores son personas mayores que participaron en el primer proyecto y se formaron en dinamización de grupos.

Y el tercer proyecto, denominado Juegos de memoria, es de autogestión; se orienta a personas que desean entrenar su memoria a través de la promoción de la autonomía y la autodisciplina. Es un proyecto con poca estructura formal y su realización está condicionada por las necesidades expresadas por las propias personas.

Los contenidos del proyecto se estructuran en cuatro bloques. En el primero, se define la memoria, su estructura y cómo se produce el acto de memorización. En el segundo, se focaliza en la atención como función cognitiva que tiene una notable incidencia en el proceso de la memorización. En el tercero, se centra la atención en el proceso de envejecimiento y cómo este afecta a la memoria. En este mismo bloque se trabajan los mitos y prejuicios entorno a esta facultad cognitiva en personas adultas y mayores con el objetivo de tomar conciencia de estas falsas creencias. Y en el último, se aborda el entrenamiento de la memoria proponiendo distintos tipos de actividades que pueden realizarse para favorecer dicho proceso y estrategias de memorización.

La metodología del proyecto se sustenta en dos elementos: el grupo y la interrelación entre iguales. Se parte del trabajo en grupo por ser una herramienta enriquecedora y estimulante. La interrelación entre iguales facilita la ayuda mutua y la disminución de la ansiedad ocasionada por la pérdida subjetiva de la memoria; los participantes tienen espacios para intercambiar sentimientos y vivencias, hecho que facilita trabajar el segundo objetivo.

Las actividades lúdicas, su progresivo aumento del grado de dificultad, la combinación del trabajo individual con el grupal y la intervención multifactorial son otros elementos metodológicos del proyecto.

Para evaluar el proyecto se establecieron siete indicadores: la adquisición de los contenidos, la aplicabilidad de los contenidos trabajados en el aula a la vida cotidiana, la identificación de las pérdidas propias del proceso de envejecimiento saludable, el grado de asistencia, el nivel de participación en el aula, el esfuerzo y la constancia del participante y el grado de interrelación y comunicación entre los participantes.

La duración del proyecto es de doce sesiones de noventa minutos cada una. El educador debe realizar principalmente dos tareas. Una primera es la dinamización, facilitar la interrelación y la buena comunicación entre los miembros del grupo para promocionar el aprendizaje y la ayuda mutua. La segunda tarea consiste en incentivar a los participantes para que realicen las actividades de aprendizaje dentro y fuera del aula con el fin de promover la transferencia de conocimientos y la reutilización de estrategias de memorización como la repetición.

El proyecto Practica tu memoria tiene similitudes a nivel educativo con el proyecto Entrenamiento de memoria, ya que los contenidos a tratar, los indicadores de evaluación y la estructura de las sesiones son iguales. A nivel organizativo también presenta semejanzas: la duración de las sesiones. La diferencia entre estos dos proyectos son los objetivos, que son: entrenar la memoria, compartir con los compañeros las inquietudes y dificultades del entrenamiento de la memoria en el día a día, promocionar la ayuda mutua y potenciar el voluntariado entre los participantes para favorecer el envejecimiento productivo, recordar conocimientos y estrategias trabajados en el programa Entrenamiento de memoria. Un elemento claramente diferenciador entre los dos proyectos es que los participantes del segundo participaron anteriormente en el primero.

El dinamizador es una persona mayor voluntaria que previamente participó en el proyecto Entrenamiento de la memoria y posteriormente realizó una formación de dinamización de grupos. Su tarea consiste en motivar a los participantes, que también han sido participantes del primer proyecto, y reforzar el entrenamiento.

\section{Necesidad de medir la transferencia}

El programa Si entrenamos, recordamos nace en el 2006 con la elaboración del diseño. Se implementó y evaluó en el año siguiente y en el 2009 se publicó (VidalMartí, 2012). La necesidad de innovar y de adaptarse a la realidad conllevó una nueva reflexión del programa y se establecieron cambios para sistematizar mucho más la intervención. Es a partir de estas modificaciones que los investigadores y educadores que imparten este programa se plantean la necesidad de medir la evaluación de la transferencia.

Se entiende por evaluación de la transferencia el proceso que «determina el grado en que los participantes aplican en su puesto de trabajo los aprendizajes y las competencias alcanzadas con la formación» (Pineda, 2011, p. 122).

La finalidad de medir la transferencia es para garantizar que los participantes que finalizaron con éxito el primer proyecto, y deseaban implicarse en el segundo como dinamizadores, dispusieran de las competencias básicas y necesarias para que fuera posible.

Se consultaron distintos modelos teóricos y se escogió el modelo de evaluación de Kirkpatrick por su claridad conceptual. Según este autor, la evaluación de la transferencia se estructura en cuatro niveles: reacción, aprendizaje, comportamientos/conductas y resultados. Si la medición de los cuatro niveles es positiva y satisfactoria se produce transferencia. A continuación, se definen cada uno de estos niveles (Kirkpatrick, 1996; 1999).

En relación con el primer nivel, la reacción, consiste en medir el nivel de satisfacción del participante. Conocer la valoración que realiza el participante de la 
acción formativa posibilita conocer aspectos valorativos de cada participante $y$, a su vez, se hace una media de cada ítem, que permite saber qué aspectos gustaron y cuáles no, lo que posibilita a los educadores valorar si es recomendable hacer cambios para las próximas ediciones.

Según Kirkpatrick, si la formación es eficaz, los participantes reaccionarán favorablemente al segundo nivel: el aprendizaje. Consiste en medir la adquisición de conocimiento aprendido durante el proyecto por parte de los participantes. Este nivel ofrece información en relación con el grado de conocimiento adquirido y su transferencia (Rodríguez, 2005).

En relación con el tercer nivel, los comportamientos/ conductas, se trata de medir los cambios en las pautas de comportamiento. Estos cambios pueden producirse dentro y/o fuera del espacio formativo. Es conveniente centrarse en la medición de los cambios que se producen en el espacio formativo.

$\mathrm{Y}$ en cuanto a los resultados, consiste en medir los efectos que el proyecto genera en las distintas áreas de la organización. Este efecto es lo que se conoce como impacto y se mide con relación a los beneficios económicos que la formación conlleva a la organización.

En definitiva, si los resultados de la reacción, el aprendizaje, los comportamientos y las conductas son positivos se producirá transferencia de conocimiento.

El objetivo de esta investigación era evaluar la transferencia de los conocimientos del educador voluntario, que en el primer caso fue participante y en el segundo dinamizador.

\section{Método}

\section{Participantes}

En un principio, la muestra estaba formada por 76 participantes, tres de los cuales quedaron excluidos por no querer participar en la investigación y 44 por desear solo participar en el proyecto Entrenamiento de la memoria y no ser dinamizadores del segundo proyecto Practica tu memoria. La muestra final fue de 29 participantes.

En el estudio, participaron 7 hombres y 22 mujeres. La edad media fue de 74,7 años, de un rango comprendido entre los 62 y 77 años. Los participantes eran de la ciudad de Barcelona y grandes poblaciones cercanas a esta ciudad. No presentaban deterioro cognitivo.

Para establecer la selección de la muestra se determinaron criterios de inclusión y exclusión. Los dos criterios de inclusión fueron: participar en el proyecto Entrenamiento de la memoria y manifestar quejas subjetivas de memoria. Los criterios de exclusión fueron tres: tener menos de 60 años, presentar deterioro cognitivo y ser voluntario del proyecto Practica tu memoria.

Todo participante, antes de empezar el proyecto Entrenamiento de la memoria, participó en un proceso selectivo que consistió en una entrevista individual y la administración de un test de screening (Short Portable Mental Status) (Martínez de la Iglesia et al., 2001), para identificar si la persona presenta o no deterioro cognitivo. Mediante este proceso, el educador pudo saber el grado de interés, el nivel de quejas subjetivas de memoria, su nivel de instrucción y si presentaba o no deterioro cognitivo. Si algún participante contestó puntuaciones por debajo de las establecidas en el test, se recomendó a la persona no participar en el programa por no ser un recurso adecuado.

\section{Material}

Los instrumentos utilizados fueron cuatro: cuestionario de satisfacción, prueba de conocimientos, checklist y entrevista.

El cuestionario de satisfacción consta de un ítem que valora globalmente el proyecto y doce ítems específicos. El cuestionario se estructura en cuatro partes: en la primera, se pide al participante que escriba la fecha y el lugar de realización del proyecto; en la segunda, se valoran los doce aspectos específicos del proyecto (contenidos, metodología, expectativas, organización, instalaciones, duración, horario, número de sesiones, materiales, interés y dedicación del educador, puntualidad y compañerismo y clima de grupo) a través de una escala tipo likert de diez valores, en la que 1 significa baja puntuación y 10 excelencia. En la tercera parte, se valora el proyecto en términos generales utilizando la misma escala likert que en la segunda, y en la cuarta hay un espacio abierto para las observaciones.

La prueba de conocimientos consta de dos partes diferenciadas: una primera donde cada participante debe responder a los catorce enunciados de manera dicotómica (cierto/falso); y una segunda que consta de tres preguntas abiertas. Los enunciados de la primera parte son conocimientos que parten de los mitos y prejuicios de Higbee (2008). Este autor afirma que hay una serie de creencias erróneas sobre la memoria que influyen en el proceso de memorización de las personas mayores. La finalidad de utilizar estos mitos es saber si desaparecen las ideas preconcebidas de la memoria después de haber participado en el primer proyecto.

Se utilizaron dos checklists: el de observación de los cambios de comportamiento y el de aplicabilidad. El de observación consta de siete ítems que se valoran con un sí o un no, y estos son: 1) grado de atención, 2) realización de las actividades en el aula, 3) realización de las actividades en casa, 4) puntualidad, 5) participación en el aula 6) participación con los compañeros y 7) con los educadores.

El checklist de aplicabilidad es para saber si los participantes del proyecto Entrenamiento de la memoria explican y transmiten a los participantes de Practicar la memoria los contenidos y las estrategias. Consta de cuatro ítems que se valoran de manera dicotómica (sí/ no), y estos son: explica los contenidos, su exposición es clara y comprensible, el tipo de actividades que realiza facilitan el aprendizaje y sabe resolver las preguntas que plantean los participantes.

La entrevista del educador consta de cuatro preguntas. Cada pregunta responde a un ítem del checklist de 
aplicabilidad. La finalidad de la entrevista es verificar los resultados obtenidos en el checklist.

\section{Procedimiento}

El procedimiento de esta investigación descriptiva y de carácter exploratorio siguió tres fases. La primera consistió en presentar la investigación a los responsables de las organizaciones donde se llevó a cabo el programa y a los educadores, con el fin de conocer el interés de la investigación. La segunda fase, la recogida de datos, se dividió en dos momentos diferenciados. Un primero que correspondió a la finalización del primer proyecto y un segundo que se realizó al cabo de seis meses, mientras los participantes dinamizaban el segundo proyecto; este periodo corresponde al tiempo organizativo necesario para formar a los participantes del primer proyecto como dinamizadores e iniciar el segundo proyecto.

Con el fin de garantizar la voluntariedad de los participantes, se decidió explicarles la investigación con el objetivo de preguntar quién deseaba participar. Los datos recogidos en el primer momento fueron el último día del primer proyecto por parte del educador de referencia para no interferir en su desarrollo; y los de la segunda fase se recogieron en dos momentos distintos: cuando se realizó la observación a medio proyecto y al final realizando las entrevistas a los educadores. La tercera fase consistió en construir la matriz, introducir los datos y hacer su análisis mediante el paquete estadístico SPSS versión 19.

\section{Resultados}

A continuación se presentan los resultados de la evaluación de la transferencia del programa de memoria.

La puntuación del cuestionario de satisfacción más baja fue la duración con un 4,49 de media (véase tabla 1). Las instalaciones (6.22), el compañerismo (6.40), la organización (6.49) y el número de sesiones (6.85) se valoran de media con un 6; los materiales (7.53) y el horario (7.95) con un 7; la metodología (8.67), los contenidos (8.76) y las expectativas (8.99) con un 8; y

Tabla 1. Resultados obtenidos del cuestionario de satisfacción

\begin{tabular}{lll}
\hline & Media & Desviación típica \\
\hline Contenidos & 8.76 & .880 \\
Metodología & 8.67 & 1.173 \\
Expectativas & 8.99 & 1.306 \\
Organización & 6.49 & 1.308 \\
Instalación & 6.22 & 1.426 \\
Duración & 4.49 & 1.344 \\
Horario & 7.95 & .932 \\
Número de sesiones & 6.85 & 1.752 \\
Materiales & 7.53 & 1.920 \\
Interés del educador & 9.12 & 1.142 \\
Puntualidad & 9.26 & .910 \\
Compañerismo & 6.40 & 1.433 \\
Valoración global & 9.73 & .758 \\
\hline
\end{tabular}

el interés del educador (9.12), la puntualidad (9.26) y la valoración global (9.73) con un 9. La disparidad de puntuación entre los participantes se observa en los materiales (DT=1.920); en cambio, la valoración global (DT=.758) fue el ítem con una mayor unanimidad.

En relación con la prueba de conocimientos, cabe comentar que todos los participantes obtuvieron puntuaciones superiores a 9. Este prueba se puntuó de 0 a 17; si un participante había respondido todas las respuestas de manera incorrecta la puntuación era un 0; en cambio, era un 17 cuando todas eran correctas. La puntuación de 15 (40.69\%) fue la más predominante y la de 12 la menos relevante, con un $0 \%$. La media de la puntuación de la prueba de conocimiento fue un 14,95 sobre 17 y su desviación típica 1,878.

La puntuación más baja del checklist de observación fue el ítem puntualidad con un 0.69 de media y el grado de atención con un 0.93 la más alta (véase la tabla 2). La disparidad de puntuación entre los participantes se observa en la puntualidad (DT $=.471)$; en cambio, el grado de atención (DT=.258) fue el ítem con una mayor unanimidad.

En relación al checklist de aplicabilidad, el 89.7\% de los participantes supo explicar los contenidos de manera clara y comprensible; el 93.1\% realizó actividades que facilitaban el aprendizaje y el 69\% supo responder las dudas planteadas por los participantes del proyecto Practica tu memoria. El ítem con una mayor unanimidad fue el tipo de actividades que facilitaban el aprendizaje $(\mathrm{DT}=.258)$ y saber responder las dudas $(\mathrm{DT}=.471) \mathrm{el}$ que menos.

Los resultados de las entrevistas son concordantes con los del checklist de aplicabilidad.

\section{Discusión}

El objetivo de este estudio era conocer la transferencia que tiene el programa de memoria Si entrenamos, recordamos. Para ello, se analizaron los resultados de satisfacción, adquisición de conocimientos, cambios comportamentales y aplicabilidad. De acuerdo con dichos resultados, el programa tiene una transferencia en la población a la cual se dirige. La evidencia de ello son las altas puntuaciones de los resultados del checklist de aplicabilidad y la confirmación de dichos resultados por parte de los educadores.

Según el modelo de Kirkpatrick, hay transferencia de conocimiento cuando los participantes aplican los

Tabla 2. Resultados obtenidos del checklist de cambios de conducta

\begin{tabular}{lll}
\hline & Media & Desviación típica \\
\hline Grado atención & .97 & .186 \\
Realización actividades aula & .86 & .351 \\
Realizar deberes & .93 & .258 \\
Puntualidad & .69 & .471 \\
Participación aula & .90 & .310 \\
Participación con los compañeros & .72 & .455 \\
Participación con los educadores & .93 & .258 \\
\hline
\end{tabular}


conocimientos y aprendizajes en el lugar de trabajo. En este estudio, esto significa que los participantes que han asistido en el primer proyecto transmiten a los participantes del segundo, Practica tu memoria, los conocimientos y aprendizajes adquiridos. La transferencia se produce porque 9 de cada 10 participantes expresan que los contenidos fueron explicados de manera clara y comprensible por los dinamizadores, y 7 de cada 10 respondieron que los últimos sabían responder sus dudas. Este segundo resultado, que es ligeramente inferior al primero, se explica por la interferencia de prejuicios y creencias erróneas entorno a la memoria -aunque dicho conocimiento, que se trabaja en la formación, sea resistente al cambio- o por otros motivos no relacionados con los contenidos (edad similar entre dinamizado y participantes, etc.).

Este hallazgo no puede contrastarse con otros estudios de índole similar porque esta investigación es exploratoria, y no se encontró hasta la fecha ninguna investigación que evalúe la transferencia de los programas de entrenamiento de la memoria. Las investigaciones encontradas se dirigen a programas formativos, pero no se encontró ninguno relacionado con la población mayor (Tejada Fernández et al., 2008; Gairín Sallán, 2010; Feixas et al., 2013; Fernández Díaz, 2013; Garello \& Rinaudo, 2013 Pineda \& Quesada, 2013)

Con relación a los programas de entrenamiento de la memoria, existen estudios para conocer su eficacia y utilidad. Montejo (2003) estableció que un 77\% de los participantes mejoró de manera satisfactoria su percepción de entrenamiento de la memoria y observó un descenso notable de las quejas subjetivas que presentaban. A su vez, este mismo estudio constató una mejoría del estado de ánimo de las personas que participaban en dichos programas.

Otros estudios (Calero García \& Navarro-González, 2005; Auffray \& Juhel, 2001) han determinado que la participación de las personas mayores en programas de memoria contribuye a mejorar su rendimiento cognitivo, y corroboró dicho fenómeno en un meta análisis (Verhaeghen, Marcoen \& Goossens, 1995). StigsdotterNeely y Backman (1995) establecieron que participar en dichos programas favorecía que el rendimiento de la memoria se mantuviera durante seis meses.

Todas estas investigaciones permiten conjeturar con los resultados obtenidos de esta investigación. Si los participantes que asisten a los proyectos de entrenamiento de la memoria tienen una mayor percepción de su funcionamiento, esto incide de manera positiva en la adquisición de los contenidos. Si los participantes, una vez han finalizado el proyecto Entrenamiento de la memoria optan a ser dinamizadores de Practica tu memoria disponen de los conocimientos adquiridos en el primer proyecto para transferirlos en el segundo.

\section{Conclusiones}

Los resultados obtenidos de la investigación muestran que el programa de memoria conlleva una transferencia de conocimientos en la población diana. Las altas puntuaciones del checklist de aplicabilidad, los resultados confirmatorios de la entrevista y la adquisición de los conocimientos son tres factores que corroboran dicha afirmación.

Estos resultados conllevan tres conclusiones. Una primera es el aumento de bienestar de los participantes en el programa de memoria. Los participantes expresaron un alto grado de satisfacción por haber participado y esto facilita una mayor motivación para el aprendizaje y, por consiguiente, por la transferencia. Una segunda es incrementar el sentimiento de utilidad. El participante, al saber que puede ser dinamizador, puede sentirse emocionalmente y socialmente mejor porque tiene la oportunidad de aportar su quehacer a otras personas. Esta situación es positiva para la persona e incrementa su motivación, hecho que lleva a la persona a ser dinamizador y repercute de manera positiva en la transferencia. Y una tercera es el impacto; es decir, la disminución del coste para la organización. La organización forma a participantes, y los que lo desean, llegan a ser dinamizadores del segundo proyecto. La dinamización por parte de las personas mayores voluntarias disminuye el coste del programa para la entidad, al no tener que retribuir al educador profesional.

Aunque los resultados sean satisfactorios, los autores de esta investigación son conscientes de las posibles limitaciones del estudio y se identificaron dos. Una primera es la escasez de estudios. La ausencia de estudios que evalúen la transferencia de conocimientos en programas dirigidos a personas mayores conlleva que esta investigación sea exploratoria y los resultados obtenidos, aunque sean positivos, al no disponer de referentes, puede presentar sesgos metodológicos. Y una segunda es la muestra. Una muestra más amplia permitiría extraer unos conocimientos más representativos $\mathrm{y}$, por consiguiente, disponer de más matices que ayuden a valorar el programa con más detalle.

Aun así, los resultados de la investigación son realmente interesantes al tratarse de un estudio aproximativo y validan una manera distinta y diferente de concebir y realizar los proyectos de entrenamiento de la memoria.

\section{Referencias}

Auffray, C. \& Juhel, J. (2001). Effets généraux et differentiels d'un programme d'entraînement cognitif multimodal chez la personne agée. L'Année Psychologique, 101, 63-89.

Baena Díez, J. M., Riu Subirana, S. \& Martínez Adell, M. A. (2009). Evidencias sobre la utilidad de los programas de entrenamiento de la memoria. Formación médica continuada en atención primaria, 16(6), 418-423.

Calero García, M. A. \& Navarro-González, E. (2005). Eficacia de un programa de entrenamiento en memoria en el mantenimiento de ancianos con y sin deterioro cognitivo. Clínica y Salud, 17(2), 187-202.

Delgado Losada, M. L. (2015). Estudio de la eficacia de un programa de entrenamiento en estrategias para mejorar la memoria en personas mayores. Tesis doctoral. 
Feixas, M., Fernández, A., Lagos, P., Quesada, C. \& Sabaté, S. (2013). Factores condicionantes de la transferencia de la formación docente en la universidad: un estudio sobre la transferencia de las competencias docentes. Infancia y Aprendizaje, 36(3), 401-416.

Fernández Díaz, M. J. (2013). Evaluación del impacto para un cambio sostenible en las organizaciones educativas. Revista española de pedagogía, 254(71), 119-140.

Gairín Sallán, J. (2010). La evaluación del impacto en programas de formación. Revista Iberoamericana sobre calidad, eficacia y cambio en educación, 5, 8. Recuperado el 11 de julio de 2015, de http://www.rinace. net/reice/numeros/arts/vol8num5/art1.pdf

Garello, M. V. \& Rinaudo, M. C. (2013). Autorregulación del aprendizaje, feedback y transferencia de conocimiento: Investigación de diseño con estudiantes universitarios. Revista electrónica de investigación educativa, 15(2), 131-147.

Israel, L. (1992). Método de entrenamiento de la memoria. Barcelona: Semar.

Hernández Viadel, J. V. \& Latorre Postigo, J. M. (2008). Método UCLM de entrenamiento de la memoria en mayores. 102. Cuenca: Universidad de Castilla-La Mancha.

Higbee, K. (2008). Your memory: How it works and how to improve it. Boston: Da Capo Press.

Kirkpatrick, D. L. (1996). Great ideas revisited. Training and Development, 50(1), 54-65.

Kirkpatrick, D. L. (1999). Evaluación de acciones formativas: los cuatro niveles. Barcelona: Gestión 2000.

Martínez de la Iglesia, J., Dueñas Herrero, R., Onis Vilches, M.C., Aguado Taberné, C., Albert Colomer, C., \& Luque, R. (2001). Adaptación y validación al castellano del cuestionario de Pfeiffer (SPMSQ) para detectar la existencia de deterioro cognitivo en personas mayores de 65 años. Medicina clínica, 117(4), 129-134.

Montejo, P. (2001). Programas de entrenamiento de memoria. Método UMAM. Cuadernos de trabajo social, 14, 255.

Montejo, P. (2003). Programa de entrenamiento de memoria para mayores con alteraciones de memoria: resultados y predictores. Revista Española de Geriatría y Gerontología, 38(6), 316-326.

Montejo, P., Montenegro, M., Reinoso, A. I., de Andrés, M. E. \& Claver, M. D. (2001). Programa de memoria. Método UNAM. Madrid: Ayuntamiento de Madrid.

Novoa, A. M., Juárez, O. \& Nebot, M. (2008). Efectividad de las intervenciones cognitivas en la prevención del deterioro de la memoria en las personas mayores sanas. Gaceta Sanitaria, 22(5), 474-482. DOI: $10.1157 / 13126930$

Pérez, A., Roqué, M., Domènech, S., Monteserín, R., Soriano, N., Blancafort, X., Vidal, C., Petit, M., Hortal, N., Gil, C., Espelt, A\& Lopez, M.J. (2015). Efficacy of memory training in healthy communitydwelling older people: study protocol for a randomized controlled trial. BMC Geriatrics, 15(1), 113. DOI: 10.1186/s12877-015-0110-4
Pineda, P. (2011). Cómo medir el impacto de la formación: Un ejemplo del sector sanitario. Formación XXI, 18, 407-421.

Pineda, P. \& Quesada, C. (2013). Evaluación de la transferencia de la formación continua mediante el modelo ETF de factores. Revista Iberoamericana de Educación, 61(1).

Rodríguez, J. (2005). El modelo Kirkpatrick para la evaluación de la formación. Capital humano, 16,189-190.

Tejada Fernández, J., Ferrández Lafuente, E., Jurado de los santos, P., Mas Torelló, O., Navío Gámez, A. \& Ruiz Bueno, C. (2008). Implicaciones de la evaluación de impacto, Una experiencia en un programa de formación de formadores. Bordón, 60 (1), 163-185.

Stigsdotter-Neely, A. \& Backman, L. (1995). Effects of multifactorial memory training in old age: generalizability across tasks and individuals. Journal of Gerontology, 50, 134-140. DOI: 10.1093/geronb/50B.3.P134

Verhaeghen, P., Marcoen, A. \& Goossens, L. (1995). Improving memory performence in the aged throught mnemonic training: a meta-analityc study. Psychology and Aging, 7, 242-251. DOI: 10.1080/13607863.2012.667783

Vidal-Martí, C. (2012). Si entrenem, recordem. Taller de memòria per a gent gran. 2. ${ }^{\text {a }}$ edición. Barcelona: Claret.

Vidal-Martí, C. (2015). Entrena tu memoria. 100 preguntas, 100 respuestas y 100 actividades. Barcelona: Editorial Invisibles.

Vidal-Martí, C., Vilar, R., Borrell, M., Capellades, A., Carrasco, O. \& Garcia, P. (2013). Innovación en los talleres de entrenamiento de la memoria. Evolución y procesos de mejora. Revista de Intervención Educativa Social, 54, 153-163.

\section{Agradecimientos}

Gracias a Isabel, M. Carme, Núria y Francesc por su colaboración.

\section{L'avaluació de la transferència d'un programa d'entrenament de la memòria}

Resum. Els programes d'entrenament de la memòria són programes psicoeducatius dirigits a persones adultes i grans sense deteriorament cognitiu. La finalitat és entrenar la memòria $i$ optimitzar-ne el rendiment. L'objectiu d'aquest estudi és mesurar la transferència de coneixements del programa de memòria $\mathrm{Si}$ entrenem, recordem. Aquest programa s'estructura en tres projectes. Aquesta investigació se centra pròpiament a avaluar la transferència del segon projecte amb relació al primer. Es va escollir el model d'avaluació de Kirkpatrick per avaluar la transferència. En aquesta investigació de caràcter descriptiu i exploratori es van administrar quatre instruments ad hoc amb la finalitat de mesurar els quatre nivells establerts en el model Kirkpatrick. La seva administració va tenir lloc en dues etapes diferents del procés. Van participar-hi 29 persones grans. Els resultats estableixen que el $89.7 \%$ dels dinamitzadors va saber explicar els continguts, el 93\% va fer activitats facilitadores de l'aprenentatge i un $69 \%$ va respondre els dubtes plantejats pels participants. Aquests resultats van ser confirmats pels educadors.

Paraules clau: memòria; programes de transferència; transferència de la formació; gestió del coneixement 